\title{
Nachruf Prof. Dr. phil. Peter Fürstenau
}

\author{
geb. 20. Mai 1930 in Berlin, gest. 27. März 2021 in Düsseldorf
}

\section{N. Belardi}

Online publiziert: 1 . September 2021

(C) Springer Fachmedien Wiesbaden GmbH, ein Teil von Springer Nature 2021

Bis zum Jahre 2017 gehörte Peter Fürstenau dem Beirat der OSC an. Wer war er, und welches waren seine Beiträge für die Themen dieser Zeitschrift?

Nach dem Studium der Philosophie, Soziologie und Klassischen Philologie (Gräzistik) in Berlin und Frankfurt/M. wurde er mit einer Dissertation über Heidegger in Berlin promoviert und wissenschaftlicher Assistent bzw. Dozent an der damaligen PH Berlin. In Berlin absolvierte er auch eine psychoanalytische Ausbildung. Das wäre heute für einen Nicht-Mediziner bzw. Nicht-Psychologen angesichts der institutionalisierten Psychoanalyse sehr schwer möglich. 1962 kam er mit Prof. H. E. Richter, der einen Lehrstuhl sowie die Leitung der psychosomatischen Klinik erhielt, an die Universität Gießen. Dort war Fürstenau bis 1973 in verschiedenen Funktionen tätig.

In diesem Nachruf möchte ich weniger seine Leistungen für Psychoanalyse und Psychotherapie, sondern sein Wirken für Beratung, Supervision, Coaching und Pädagogik hervorheben. Wohl auch aufgrund seines ungewöhnlichen akademischen bzw. beruflichen Werdeganges steht Fürstenau am Anfang einiger neuer Sichtweisen, die alle in den Zusammenhang angewandte Psychoanalyse als Sozialwissenschaft gehören: Seine Schrift „Zur Psychoanalyse der Schule als Institution“ erschien in Kurzfassung erstmals 1964 in „Das Argument“. Es enthält ungewöhnliche kritische und weiterführende Gedanken über den Lehrerberuf und die bürokratisierte Schule. Diese stehen in der Nachfolge des damals noch vergessenen Siegfried Bernfeld. Darin finden sich auch schon Anfänge seiner späteren Organisationsanalyse bzw. Bürokratieanalyse (Institutionsberatung). Fürstenau war auch an den Anfängen der Gruppendynamik in Deutschland beteiligt. Als Student nahm ich ab Ende der

Prof. Dr. N. Belardi $(\bowtie)$

Rosenstraße 15, 51427 Bergisch Gladbach, Deutschland

E-Mail: Nando@Belardi.de 
1960er-Jahre an Selbsterfahrungsgruppen in der Universität sowie den ersten großen mehrwöchigen gruppendynamischen Laboratorien (,Organisations-Labs“) teil.

Im Jahre 1969 erfolgte Füstenaus kumulative Habilitation im Fach Psychoanalyse und Soziologie. Daraus entstanden auch das heute noch lesbare Buch „Soziologie der Kindheit“ (Heidelberg 1967) sowie Schriften über Institutionsberatung. Nun verfügte Fürstenau mit der Psychoanalyse, der Gruppendynamik und der Soziologie über verschiedene Sichtweisen, um psychisches und soziales Geschehen von Einzelnen, Gruppen, Teams und Organisationen verstehen zu können. 1970 erschien sein Beitrag über „Institutionsberatung. Ein neuer Zweig angewandter Sozialwissenschaft". Heute verwendet man eher den breiteren Begriff Organisationsberatung bzw. Organisationsentwicklung.

Wie war sein Verhältnis zu Supervision und speziell zur Teamsupervision? Laut einem Interview in „Sozialpsychiatrische Informationen“ 2/1990 bevorzugte Fürstenau bei der Veränderung von Institutionen eher die Leitungsberatung (heute würde man Coaching sagen). Denn er war der Meinung, dass die Leitungspersonen zeitweilig unterstützt werden sollten, ihre Einrichtungen so zu führen, dass dauerhafte Hilfe von außen nicht unbedingt nötig sein müsse.

Bis 1973 war er als wissenschaftlicher Rat, Professor und Abteilungsvorsteher im Zentrum für psychosomatische Medizin des Fachbereichs Humanmedizin der Universität Gießen tätig. Seine Lehrveranstaltungen wurden, wie die von Prof. Richter und anderen der Klinik, gerne von Studierenden der Gesellschafts- und Erziehungswissenschaften besucht. In diesen Fakultäten war er auch als Prüfer aktiv. In seinem 43. Lebensjahr verließ Fürstenau die sichere Beamtenstelle und ging in die Freiberuflichkeit. Künftig lebte er als Psychoanalytiker (auch Ausbilder), Psychologischer Psychotherapeut in Düsseldorf und wie man heute sagen würde, als Supervisor, Coach und Organisationsberater.

Fürstenau schrieb wenig, aber dies war gehaltvoll. Die Titel findet man im Internet. Anhänger großer Theorien, von Heilsvorstellungen oder Klein-Klein-Empirie werden enttäuscht sein. Dafür war er viel zu klug und zu pragmatisch. Ihm ging es darum, menschliche Beziehungen privat sowie beruflich zu verbessern, also um „Entwicklungsförderung durch Therapie“. Es gibt kein großes umfassendes Werk und keine „Fürstenau-Schule“. Allerdings haben Hunderte von Ärzten, Psychologen, Psychotherapeuten, Pädagogen sowie Angehörige anderer Berufsgruppen an seinen Weiterbildungsseminaren, vor allem in Venedig und in Düsseldorf, teilgenommen. Möglicherweise hatte er damit mehr bewirken können als andere, die in universitären Zusammenhängen durch umfassende Bücher bekannt geworden sind. Zuletzt sprach er von einem lösungsorientierten psychoanalytisch-systemischen Ansatz. Seine Themen kreisten um eine pragmatische Humanisierung durch Supervision, Leitungsberatung, Institutionsberatung, Alltagsthemen in der Pädagogik sowie im menschlichen Zusammenleben. Alles ist sehr lesenswert.

Über der Traueranzeige steht ein Spruch, der meiner Erfahrung nach für Peter Fürstenaus pragmatische und illusionsfreie Sicht auf das Leben ein Motto auch für andere sein könnte: „So ist das“.

\section{Nando Belardi}

